\title{
Coupling of kinetic Monte Carlo simulations of surface reactions to transport in a fluid for heterogeneous catalytic reactor modeling
}

\section{Citation for published version (APA):}

Schaefer, C., \& Jansen, A. P. J. (2013). Coupling of kinetic Monte Carlo simulations of surface reactions to transport in a fluid for heterogeneous catalytic reactor modeling. Journal of Chemical Physics, 138(5), 0541021/9. [054102]. https://doi.org/10.1063/1.4789419

DOI:

10.1063/1.4789419

Document status and date:

Published: 01/01/2013

\section{Document Version:}

Publisher's PDF, also known as Version of Record (includes final page, issue and volume numbers)

\section{Please check the document version of this publication:}

- A submitted manuscript is the version of the article upon submission and before peer-review. There can be important differences between the submitted version and the official published version of record. People interested in the research are advised to contact the author for the final version of the publication, or visit the DOI to the publisher's website.

- The final author version and the galley proof are versions of the publication after peer review.

- The final published version features the final layout of the paper including the volume, issue and page numbers.

Link to publication

\footnotetext{
General rights

- You may freely distribute the URL identifying the publication in the public portal. follow below link for the End User Agreement:

www.tue.nl/taverne

\section{Take down policy}

If you believe that this document breaches copyright please contact us at:

openaccess@tue.nl

providing details and we will investigate your claim.
}

Copyright and moral rights for the publications made accessible in the public portal are retained by the authors and/or other copyright owners and it is a condition of accessing publications that users recognise and abide by the legal requirements associated with these rights.

- Users may download and print one copy of any publication from the public portal for the purpose of private study or research.

- You may not further distribute the material or use it for any profit-making activity or commercial gain

If the publication is distributed under the terms of Article $25 \mathrm{fa}$ of the Dutch Copyright Act, indicated by the "Taverne" license above, please 


\title{
Coupling of kinetic Monte Carlo simulations of surface reactions to transport in a fluid for heterogeneous catalytic reactor modeling
}

\author{
C. Schaefer ${ }^{\mathrm{a})}$ and A. P. J. Jansen ${ }^{\text {b) }}$ \\ Eindhoven University of Technology, P.O. Box 513, 5600 MB Eindhoven, The Netherlands
}

(Received 6 November 2012; accepted 11 January 2013; published online 1 February 2013)

\begin{abstract}
We have developed a method to couple kinetic Monte Carlo simulations of surface reactions at a molecular scale to transport equations at a macroscopic scale. This method is applicable to steady state reactors. We use a finite difference upwinding scheme and a gap-tooth scheme to efficiently use a limited amount of kinetic Monte Carlo simulations. In general the stochastic kinetic Monte Carlo results do not obey mass conservation so that unphysical accumulation of mass could occur in the reactor. We have developed a method to perform mass balance corrections that is based on a stoichiometry matrix and a least-squares problem that is reduced to a non-singular set of linear equations that is applicable to any surface catalyzed reaction. The implementation of these methods is validated by comparing numerical results of a reactor simulation with a unimolecular reaction to an analytical solution. Furthermore, the method is applied to two reaction mechanisms. The first is the ZGB model for CO oxidation in which inevitable poisoning of the catalyst limits the performance of the reactor. The second is a model for the oxidation of NO on a $\mathrm{Pt}(111)$ surface, which becomes active due to lateral interaction at high coverages of oxygen. This reaction model is based on ab initio density functional theory calculations from literature. () 2013 American Institute of Physics. [http://dx.doi.org/10.1063/1.4789419]
\end{abstract}

\section{INTRODUCTION}

Many processes in chemical industry use heterogeneous catalysts, which are often supported nanoparticles of transition metals. Their activity is highly dependent on their size and shape, ${ }^{1,2}$ because the reactivity of adsorbates is different on stepped surfaces, vertices, and edges. Current microkinetic modeling methods are mostly based on the mean-field approximation, in which only one surface topology is taken into account. Moreover, in these models the lateral interactions between adsorbates at the surface are not included. Approaches that include the structure of catalysts are needed for these systems, e.g., kinetic Monte Carlo (kMC) schemes.

In reactor engineering the transport of chemical reactants and products are commonly modeled by computational fluid dynamics (CFD), which deals with transport of heat, momentum, and mass. Naturally, at each location in the reactor the reaction conditions differ; i.e., in the reactor there is a wide range of different concentrations and temperatures. These varieties of conditions give rise to different macroscopic reaction rates as boundary conditions to the CFD problem.

Proper reactor modeling needs a good description of the reactions on a catalyst's surface as provided by $\mathrm{kMC}$, and a good description of transport properties as provided by CFD. There have been many studies using kMC in which transport limitations have been ignored and CFD studies with the mean-field approximation for the surface reactions, but very few studies in which both have been combined. ${ }^{3-14}$ and only two in which a whole channel flow reactor has been

a)Electronic mail: c.schaefer@tue.nl.

b) Electronic mail: a.p.j.jansen@tue.nl. simulated. ${ }^{15,16}$ In this work we will investigate such a combined multi-scale approach and we intend to simulate reactors of large sizes (i.e., the characteristic lengths of this reactor should be larger than the mean free path of the molecules in the reactor) at steady state conditions with a molecular resolution. In CFD simulations the reaction mixture is viewed as a continuum so that these large length scales can be simulated, but it misses the molecular resolution that is needed to model the reactions on catalysts' surfaces. These small length scales can be simulated by using $\mathrm{kMC}$ simulations for surfaces with up to $10^{6}$ catalytic sites $\left(\sim 1 \mu \mathrm{m}^{2}\right)$.

In recent studies it was shown that both simulation techniques can be coupled such that the advantages of both are exploited. $^{3-16}$ In such a method the concentration of all reactive species are obtained from CFD calculations, which require the reaction rates of these species as a boundary condition. Naturally, the rates depend on the concentrations and have to be obtained from kMC simulations. The inputs and outputs of the CFD and kMC simulations are tightly coupled, so that an iterative procedure is needed wherein the concentration profile in the reactor converges to the steady state profile. This requires a large number of kMC simulations, which makes it computationally costly. In the work of Majumder et al. a gap-tooth scheme, as developed by Kevrekidis et al., was used to reduce the number of kMC simulations needed in order to simulate a pore of a membrane of which the walls were catalytically active. ${ }^{15,17}$ In this gap-tooth scheme the $\mathrm{kMC}$ simulations are performed at a limited number of locations (the teeth) in the reactor, while the reaction rates are interpolated at the locations in between (the gaps).

In our work we extend the approach by Majumder et al. such that the number of $\mathrm{kMC}$ simulations is further reduced. 
We also introduce a method to correct for possible mass balance violations. We find that such a correction is necessary because of the stochastic nature of kMC. Furthermore we present a thorough analysis of the accuracy and efficiency of the method as a function of all simulation parameters. We also apply it to models for which there is no exact analytical treatment. We even present an ab initio kinetics model of a whole reactor.

The structure of this paper is as follows. In Sec. II we introduce a method to perform mass balance corrections on the stochastic results from $\mathrm{kMC}$ simulations. We also introduce there the transport model that we solve by using CFD simulations, followed by our improved coupling method. In Sec. III this method is validated by considering a unimolecular surface reaction type. This model can be treated analytically without approximations so that we can use it to determine the accuracy of our method. In that section we also apply our method to describe a reactor wherein the reaction is described by the Ziff-Gulari-Barshad (ZGB) model, and we perform simulations on a model wherein the reaction model is based on density functional theory (DFT) calculations. We finish with Sec. IV summarizing our work and reiterating our main conclusions.

\section{METHODS}

\section{A. Surface reactions}

The surface reaction models are simulated by using kMC simulations as usual. ${ }^{18}$ The details of these models and their implementation can be found in Sec. III. We discuss here a problem that arises in the coupling with CFD due to the stochastic nature of kMC. In our work we are interested in reactors at steady state, which implies that the dynamic mass balance equation can be set to zero:

$$
0=\frac{\partial \mathbf{c}}{\partial t}=\boldsymbol{v} \times\left(\alpha \boldsymbol{\phi}^{0}\right)
$$

Here $\boldsymbol{v}$ is the stoichiometry matrix and $\alpha \boldsymbol{\phi}^{0}$ is the volumetric reaction rate, where $\alpha$ denotes the amount of catalytic surface area per unit of reactor volume and $\phi^{0}$ is the reaction rate per unit of catalytic surface area. Hence, at steady state $\boldsymbol{v} \boldsymbol{\phi}^{0}=\mathbf{0}$ should hold. The elements $v_{i j}$ of the stoichiometry matrix represent the number of elemental species $i$ (atoms or chemical groups that are unaltered during the reactions) per molecule $j$. For example, in the $\mathrm{CO}$ oxidation reaction this balance is given by

$$
\left[\begin{array}{lll}
1 & 0 & 1 \\
1 & 2 & 2
\end{array}\right]\left[\begin{array}{c}
\phi_{\mathrm{CO}}^{0} \\
\phi_{\mathrm{O}_{2}}^{0} \\
\phi_{\mathrm{CO}_{2}}^{0}
\end{array}\right]=\left[\begin{array}{l}
0 \\
0
\end{array}\right]
$$

where the first row of the stoichiometry matrix represents conservation of carbon atoms and the second row represents conservation of oxygen atoms. This implies that although there is net adsorption/desorption of molecules at the surface, the net adsorption/desorption rate of elemental species should equal zero.
During a simulation the macroscopic reaction rates fluctuate around the steady state value and the correct value is obtained by averaging over an infinite time interval. In practice only a limited number of time steps can be used, so that the mass balance is violated. This effect can be reduced by averaging over more time steps, the noise then reduces inverse proportional to the square root of the number of time steps. $^{18}$

This is a practical solution to let this error become insignificant with respect to the numerical errors due to reactor discretization and due to the usage of a gap-tooth scheme. We however feel that it is more appropriate to completely do away with this problem by performing corrections on the reaction rates, because this problem is fundamentally in contradiction with our steady state approximation. This can be illustrated in the following case. If we would have a reaction of two reactants that are fed in stoichiometric proportions to a one-dimensional reactor (see reactor description in Sec. II B) the ratio between the concentrations of these species should be equal at any position in the reactor. Independent of other numerical errors, the error in mass balance will accumulate so that significant deviations of this ratio may be found, of which the cause is usually hard to identify.

To solve this problem we developed a method to do mass balance corrections that can be applied for any chemical reaction. The rates $\phi$ obtained from $\mathrm{kMC}$ simulations are corrected with $\delta$ to new rates $\boldsymbol{\phi}^{0} \equiv \boldsymbol{\phi}+\delta$ that do obey the mass balance. There $\phi, \delta$, and $\phi^{0}$ are all vectors with components that correspond to various adsorbates. The main idea is that the correction should be as small as possible. We propose to do this by minimizing $\boldsymbol{\delta} \cdot \boldsymbol{\delta}$, constrained by expressions that follow from the mass balance.

These constraints are obtained by rewriting $\boldsymbol{v} \boldsymbol{\phi}^{0}=\mathbf{0}$ into

$$
\boldsymbol{v}^{\prime} \boldsymbol{\phi}^{0}=\mathbf{0},
$$

where $\boldsymbol{v}^{\prime}$ is the $M \times N$ sized reduced row-echelon form of $\boldsymbol{v}$, which is obtained by Gauss elimination with backsubstitution of $v{ }^{19}$ Here the zero-rows of the matrix are removed $(\operatorname{rank}(\boldsymbol{v})=M)$, and the indices of the chemical species of which the reaction rates are represented in $\phi^{0}$ are arranged such that $\boldsymbol{v}^{\prime}=[\mathbf{I} \mid \ldots]$ holds, with $\mathbf{I}$ the $M \times M$ identity matrix. Here $N$ is the number of different chemical species, and $M$ is the number of elemental species.

We now define the numerical error made due to finite time-averaging by

$$
\epsilon \equiv \boldsymbol{v}^{\prime} \phi
$$

where as discussed before, $\phi$ is the vector with reaction rates as obtained by kMC simulations. Thus, by using $\boldsymbol{\phi}^{0}=\boldsymbol{\phi}+\boldsymbol{\delta}$ we find

$$
v^{\prime} \delta=-\epsilon
$$

as constraints of the least-squares problem.

As a result of this minimization of $\delta \cdot \delta$ with respect to the vector elements $\delta_{i}$ we get

$$
\delta_{i}=-\left(\epsilon_{i}+\sum_{j=M+1}^{N} v_{i j}^{\prime} \delta_{j}\right), \quad \text { for } i \leq M,
$$


while for $i>M$ we obtain $\delta_{i}$ from solving the non-singular set of linear equations given by

$$
(\mathbf{A}+\mathbf{I}) \mathbf{u}=\mathbf{v},
$$

wherein $\mathbf{I}$ is the $N_{\text {dof }} \times N_{\text {dof }}$ identity matrix, while we define $A_{i j} \equiv \sum_{k=1}^{M} v_{k(i+M)}^{\prime} v_{k(j+M)}^{\prime}, \quad u_{i} \equiv \delta_{i+M}$ and $v_{i} \equiv \sum_{k=1}^{M} v_{k(i+M)}^{\prime} \epsilon_{k}$. Here $N_{\text {dof }}=N-M$ is the number of degrees of freedom by which the total reaction rate and the selectivity of the reaction are determined. If we, for example, again consider the $\mathrm{CO}$ oxidation reaction, we note that this reaction has a single degree of freedom by which the reaction rate is determined. The reduced row-echelon matrix form of the stoichiometry matrix is now

$$
\boldsymbol{v}^{\prime}=\left[\begin{array}{ccc}
1 & 0 & 1 \\
0 & 1 & \frac{1}{2}
\end{array}\right] .
$$

As a result, the corrected reaction rates are given by

$$
\phi_{\mathrm{CO}}^{0}=2 \phi_{\mathrm{O}_{2}}^{0}=-\phi_{\mathrm{CO}_{2}}^{0}=\frac{1}{9}\left(4 \phi_{\mathrm{CO}}+2 \phi_{\mathrm{O}_{2}}-4 \phi_{\mathrm{CO}_{2}}\right) .
$$

The approach described here can also easily be extended by including weights on the corrections $\delta_{i}$, based on the accuracy by which the corresponding $\phi_{i}$ is determined.

\section{B. Fluid phase transport modeling}

A heterogeneous catalyzed reactor often contains porous pellets that consist of a supporting material at which catalytic nanoparticles are deposited. In practice effective transport parameters are experimentally determined based on continuum transport models, so that these can be used directly in continuum reactor models. ${ }^{20}$ These models can incorporate much detail, such as velocity profiles and axial and radial dispersion, but at cost of having to introduce more parameters. Since we are primarily interested here in the coupling between CFD and $\mathrm{kMC}$, we restrict ourselves for simplicity to cases of a tubular reactor wherein transport is fast with respect to the reaction rate, so that radial dispersion can be neglected. Further we assume a flat velocity profile and low concentrations of reactant in a carrier fluid. With these assumptions we do not have to solve the Navier-Stokes equations or the heat equation, and we may invoke a one-dimensional mass transport model wherein the concentrations and reaction rate only vary in the axial direction of the reactor. ${ }^{21}$ The design equation of the reactor is then given by ${ }^{22}$

$$
v \frac{d c_{i}}{d z}=\alpha \phi_{i}
$$

wherein $v$ is the velocity of the fluid, $c_{i}$ is the concentration of species $i, z$ is the position in the reactor, $\alpha$ is the amount of reactive surface area per amount of reactor volume, and $\phi_{i}$ is the desorption minus the adsorption rate of species $i$ per unit of catalytic surface area. At steady state $\phi_{i}$ is also equal to the formation rate. This equation is numerically solved by using the explicit Euler method, which has a first order accuracy in $z .^{19}$

Naturally, Eq. (10) may be too simple for some practical applications, as it does not describe nonlinear effects such as moving fronts, oscillations, and bistabilities. Currently, such problems are dealt with in more sophisticated reactor models in which one makes use of a mean field approximation for the reactions. We could use the methods that are used there to deal with these effects also in our approach. After the mass balance corrections and the removal of the noise our approach essentially only replaces the mean field part with the better kMC simulations. The reason we use Eq. (10) instead of a more sophisticated model for the transport is that we want to focus on the coupling of $\mathrm{kMC}$ with the transport model and not to be distracted by problems originating from nonlinear effects.

\section{Coupling}

The reactor is modeled with a finite difference grid, of which the grid points represent positions in the reactor that are associated with a velocity field, concentrations, and a temperature. As shown in Fig. 1, some grid points are located at a catalytic surface, where reactions take place and the rate of this reaction is a boundary condition of the CFD calculation. In our one-dimensional case every grid point is associated with a reaction rate. If we would perform KMC simulations for each of these grid points this would be a very costly computational task. For that reason the number of kMC simulations is reduced by invoking a gap-tooth scheme. ${ }^{17}$ In this scheme we evenly space the teeth where kMC simulations are done and we perform linear interpolation of the reaction rates as a function of the position in the gaps. This allows us to use the upwinding scheme in Fig. 2. In this scheme the concentration at the inlet of the reactor is given. From this the reaction rate is calculated using $\mathrm{kMC}$, while the concentration and rate at the next tooth are estimated using

$$
c_{i}^{k}=c_{i}^{k-1}+\left(\frac{\alpha \phi_{i}}{v}\right)^{k} \Delta z,
$$

where $\Delta z$ is the distance between grid points. They are numbered starting at the inlet. These numbers are the subscripts in Eq. (11). The adsorption rates that determine $\phi_{i}^{k}$ are dependent on $c_{i}^{k}$. This means that the equation has to be solved iteratively to become self-consistent. This is done by first estimating the rates $\phi_{i}^{k}$ at the next tooth and using linear interpolation for the grid points between the teeth. Equation (11) is
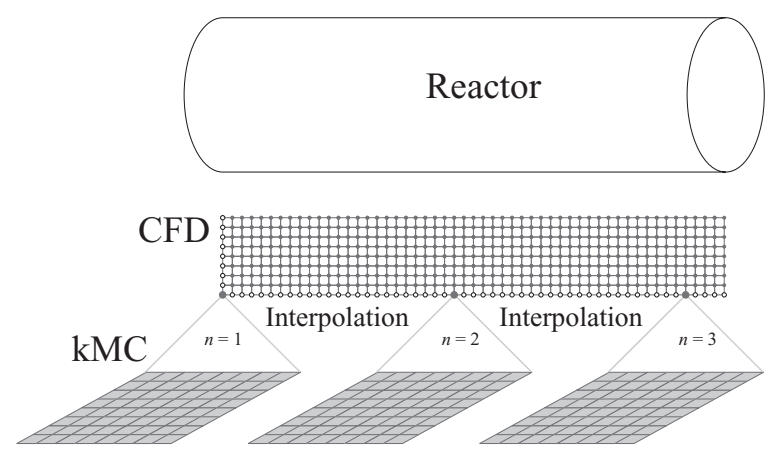

FIG. 1. We discretize a reactor in a CFD grid, of which the boundaries are given by the concentrations at the inlet and the reaction rates at the catalytic surface. The reaction rates are determined by performing kMC simulations only at certain grid points (the teeth, indexed by $n$ ) and interpolation is used in the gaps to reduce the number of $\mathrm{kMC}$ simulations. 


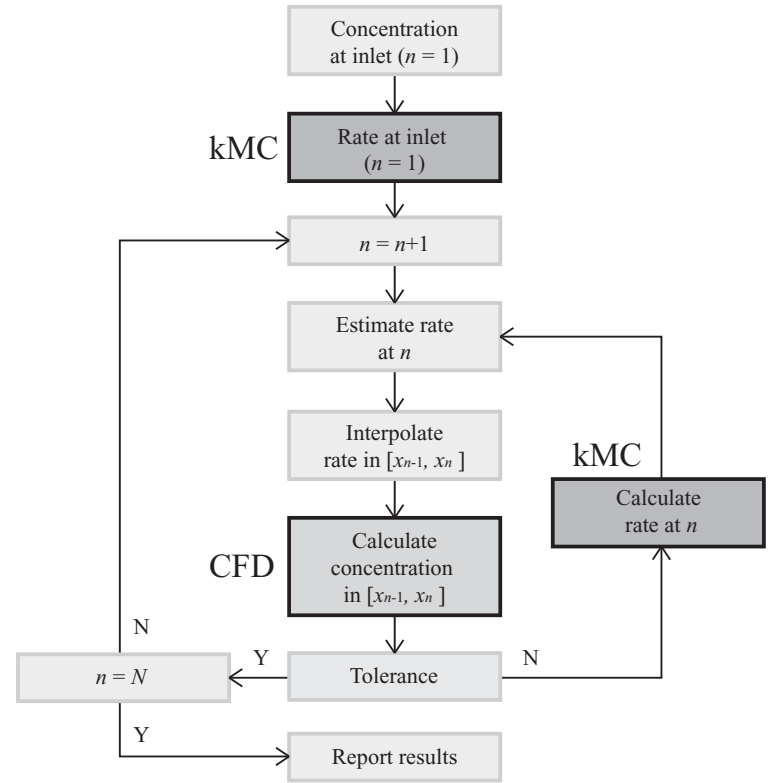

FIG. 2. The reactor is solved stepwise by using an iterative procedure between two teeth at positions $x_{n-1}$ and $x_{n}$ to determine the reaction rates and concentrations. After convergence we move to the next tooth and solve this part of the reactor with the same procedure.

then used to get the concentrations. The new rates at the next tooth can then be computed by $\mathrm{kMC}$, after which the procedure is repeated starting with interpolation and the calculation of new concentrations. To ensure stability

$$
\Delta z \leq 2 \min _{i}\left(\left[\frac{\partial}{\partial c_{i}} \frac{\alpha \phi_{i}}{v}\right]^{k}\right)^{-1}
$$

should hold. ${ }^{19}$ This essentially states that there should not be too much curvature of the concentration between two grid points. This we prevented in first place for the gap-tooth scheme to be applicable, as there should not be too much curvature between the teeth. Thus, this stability criterion is particularly of importance in other systems, e.g., tubular reactors with Hagen-Poiseuille flow. In such reactors the flow velocity at the wall is zero so that at the wall a very fine discretization is needed, and one should use an adaptive mesh and unevenly spaced teeth. Rather than a stability criterion, we should introduce only small concentration changes in each upwinding step to keep the discretization error small. From Eq. (11) it can be seen that we should have

$$
\Delta z \ll \min _{i}\left[\left(\frac{v}{\alpha\left|\phi_{i}\right|}\right)^{k} c_{i}^{k-1}\right]
$$

Our approach is different from the approach used by Majumder et al. ${ }^{15}$ in which all concentrations and all rates are updated at the same time. That approach has the advantage of a smooth interpolation technique in which curvature is taken into account, but it requires more $\mathrm{kMC}$ simulations because in the first iterations the estimates for the rates and concentrations far in the reactor are very poor.

\section{RESULTS AND DISCUSSION}

\section{A. Validation: A unimolecular surface reaction}

To validate the implementation of our method we consider a case in which the kMC results can be compared to exact analytical results. These should be the same except for errors caused by the stochastic nature of $\mathrm{kMC}$ and the discretization of the reactor. We take a unimolecular reaction in which all elementary reaction steps take place at a single catalytic site and the adsorbates have no lateral interactions with neighboring adsorbates. The steps are shown in the reaction scheme below.

$$
\begin{gathered}
\mathrm{A}+* \underset{k_{\mathrm{A}}^{-}}{\stackrel{k_{\mathrm{A}}^{+}}{\rightleftharpoons}} \mathrm{A}_{\mathrm{ads}}, \\
\mathrm{A}_{\mathrm{ads}} \underset{k^{-}}{\stackrel{k^{+}}{\rightleftharpoons}} \mathrm{B}_{\mathrm{ads}}, \\
\mathrm{B}_{\mathrm{ads}} \underset{k_{\mathrm{B}}^{+}}{\stackrel{k_{\mathrm{B}}^{-}}{\rightleftharpoons}} \mathrm{B}+* .
\end{gathered}
$$

Provided that no molecules B are supplied to the reactor at its inlet, it can be shown that the formation rate $\phi_{i}$ at the surface is given by

$$
-\phi_{B}=\phi_{A}=\frac{-\kappa_{1}+\kappa_{2} X}{\kappa_{5} / c_{\mathrm{A}}^{0}+\kappa_{3}+\kappa_{4} X} .
$$

In this equation $X \equiv 1-c_{\mathrm{A}} / c_{\mathrm{A}}^{0}$ is the conversion with $c_{\mathrm{A}}^{0}$ the concentration of $\mathrm{A}$ at the inlet. ${ }^{22}$ The concentrations of $\mathrm{A}$ and $\mathrm{B}$ are related to $X$ via $c_{\mathrm{A}}=(1-X) c_{\mathrm{A}}^{0}$ and $c_{\mathrm{B}}=X c_{\mathrm{A}}^{0}$, respectively. Furthermore we have defined

$$
\begin{gathered}
\kappa_{1} \equiv k^{+} k_{\mathrm{B}}^{-} k_{\mathrm{A}}^{+}, \\
\kappa_{2} \equiv k^{+} k_{\mathrm{B}}^{-} k_{\mathrm{A}}^{+}+k^{-} k_{\mathrm{B}}^{+} k_{\mathrm{A}}^{-}, \\
\kappa_{3} \equiv\left(k^{-}+k^{+}+k_{\mathrm{B}}^{-}\right) k_{\mathrm{A}}^{+}, \\
\kappa_{4} \equiv\left(k^{-}+k^{+}+k_{\mathrm{A}}^{-}\right) k_{\mathrm{B}}^{+}-\left(k^{-}+k^{+}+k_{\mathrm{B}}^{-}\right) k_{\mathrm{A}}^{+}, \\
\kappa_{5} \equiv\left(k_{\mathrm{A}}^{-}+k^{+}\right)\left(k_{\mathrm{B}}^{-}+k^{-}\right)-k^{-} k^{+} .
\end{gathered}
$$

With this the design equation of the reactor in Eq. (10) can be solved analytically, giving

$$
\frac{\alpha}{c_{\mathrm{A}}^{0} v} z=\frac{\left(\kappa_{5} \kappa_{2} / c_{\mathrm{A}}^{0}\right)+\kappa_{2} \kappa_{3}+\kappa_{1} \kappa_{4}}{\kappa_{2}^{2}} \ln \left(1-\frac{\kappa_{2}}{\kappa_{1}} X\right)+\frac{\kappa_{4}}{\kappa_{2}} X .
$$

As expected, at $z=0$ no reactant is converted $(X=0)$ while for an infinitely long reactor $z \rightarrow \infty$ the reactor mixture will reach thermal equilibrium $X \rightarrow \kappa_{1} / \kappa_{2}$ so that $c_{B} / c_{A} \rightarrow\left(k_{\mathrm{A}}^{+} k^{+} k_{\mathrm{B}}^{-}\right) /\left(k_{\mathrm{A}}^{-} k^{-} k_{\mathrm{B}}^{+}\right)$.

The reaction rates and concentration at the inlet of the reactor have been chosen the same as in the work of Majumder et al. ${ }^{15}$ Also the dimensions of the reactor have been taken from that publication, except that the length has 

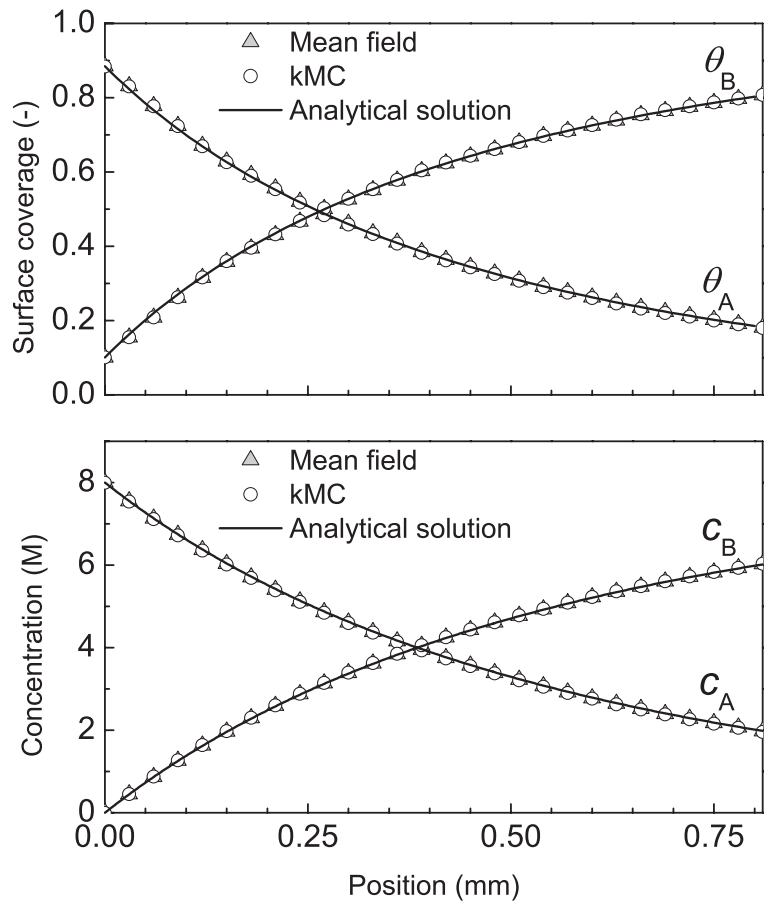

FIG. 3. Coverages and concentrations as a function of the position for the unimolecular model. These were calculated analytically and numerically with 401 grid-points and 401 teeth (which were varied in Fig. 4) wherein the rates were calculated by both the mean field model and by kMC simulations. In the $\mathrm{kMC}$ simulations a $128 \times 128$ square grid was used and simulated for $0.1 \mathrm{~s}$.

been increased to get a higher conversion. We have chosen the flow velocity to be $0.01 \mathrm{~m} \mathrm{~s}^{-1}$ and $\alpha=2 \times 10^{8} \mathrm{~m}^{-1}$. The numerical results have been obtained by using both the mean field model and kMC simulations with a $128 \times 128$ grid and a simulation length of $0.1 \mathrm{~s}$. For each simulation the final configuration of the previous simulation was used as input to reduce the number of $\mathrm{kMC}$ steps needed to equilibrate. The CFD grid has 401 grid points. Initially kMC simulations were performed at each point, i.e., no gap-tooth. The numerical solutions (concentrations and surface coverages as a function of the position) match the analytical values in Fig. 3 perfectly, from which we can conclude that our implementation is correct.

As a next step we have studied the influence of the numerical settings on the accuracy of the results. First, the grid den-

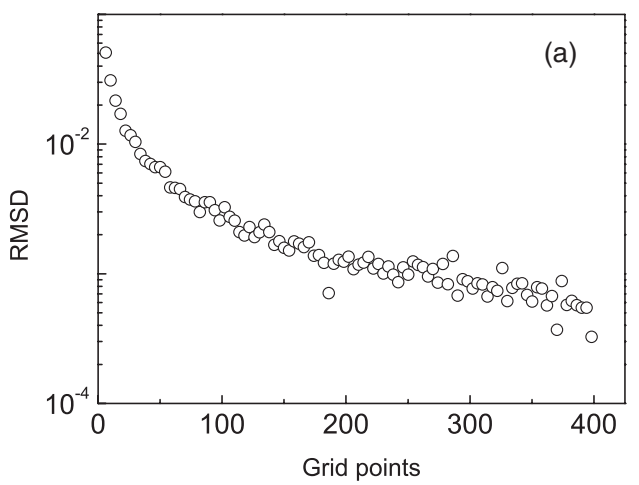

sity has been varied but no gap-tooth scheme has been used. Second, the number of grid points was kept constant at 401 while the number of teeth was varied. To probe the error we use the root-mean-square deviation, defined by

$$
\mathrm{RMSD} \equiv \sqrt{\frac{1}{N_{\text {grid }}} \sum_{i=1}^{N_{\text {grid }}}\left(X_{i}-X\left(z_{i}\right)\right)^{2}}
$$

where $N_{\text {grid }}$ is the number of grid points used in the simulation, $X_{i}$ is the conversion at grid point $i$ determined in the numerical scheme, and $X\left(z_{i}\right)$ is the conversion at the same position as determined by solving Eq. (17). As one would expect, increasing the grid density decreases the error as can be seen in Fig. 4(a). However, Fig. 4(b) shows that the number of teeth can be reduced substantially without affecting the error. We have found that for this reactor simulation an acceptable error of $3 \times 10^{-3}$ can be obtained by using only 100 grid points and $10-15$ teeth. We typically need 2 or 3 iterations to get steady state, so that we need to do about $40 \mathrm{kMC}$ simulations. This should be compared with the method of Majumder et al. in which only 6 teeth are used but with 20 iterations for a total of $120 \mathrm{kMC}$ simulations.

\section{B. ZGB model for CO oxidation}

The ZGB model for CO oxidation is a simple model with three irreversible reaction steps. In spite of its simplicity it shows two phase transitions from a reactive phase to a $\mathrm{CO}$ and to a $\mathrm{O}$ poisoned state. ${ }^{23}$ When evaluated with a mean field model there is no $\mathrm{O}$ poisoning, so that it is necessary to use $\mathrm{kMC}$ simulations. In these simulations vacant sites are places where $\mathrm{CO}$ can adsorb with probability proportional $W_{\mathrm{CO}}$ and if two vacant sites are neighboring $\mathrm{O}_{2}$ can adsorb with a probability proportional to $W_{\mathrm{O}_{2}}$.

$$
\mathrm{CO}+* \stackrel{W_{\mathrm{CO}}}{\rightarrow} \mathrm{CO}_{\mathrm{ads}}
$$

$$
\mathrm{O}_{2}+2 * \stackrel{W_{\mathrm{O}_{2}}}{\rightarrow} 2 \mathrm{O}_{\mathrm{ads}}
$$

$$
\mathrm{CO}_{\mathrm{ads}}+\mathrm{O}_{\mathrm{ads}} \stackrel{\infty}{\rightarrow} \mathrm{CO}_{2}+2 *
$$

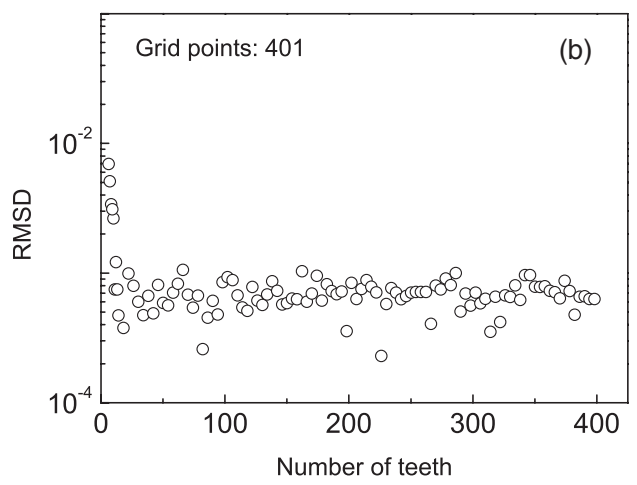

FIG. 4. The root-mean-square deviations (RMSD) of the kMC simulations of Fig. 3 with variable numerical settings. (a) The number of grid points is varied but no gap-tooth scheme is used. (b) The number of grid points is fixed at 401 while the number of teeth, at which the actual reaction rate calculations take place, is varied. 


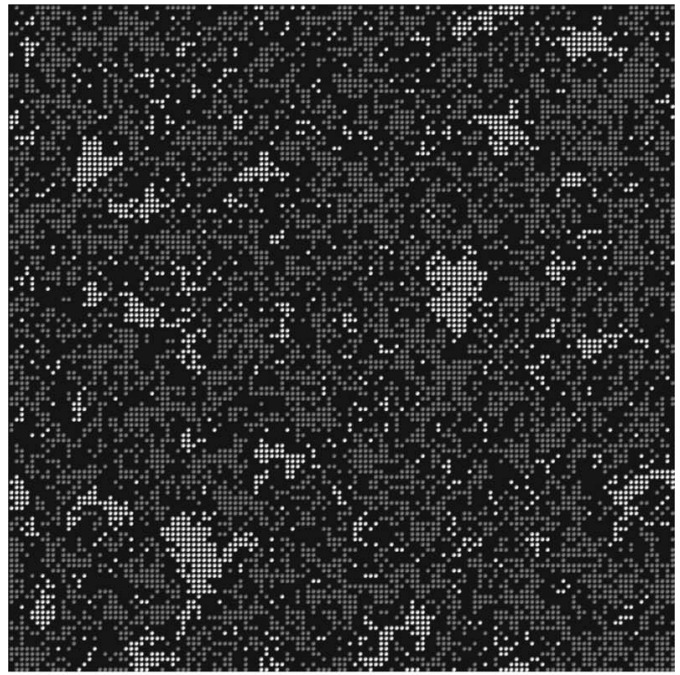

(a)

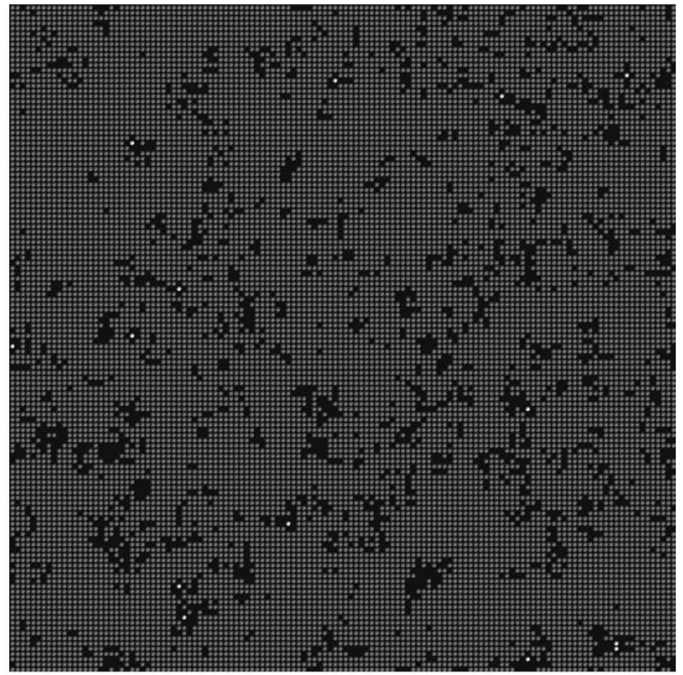

(b)

FIG. 5. Configurations of adsorbates at the catalytic surface at the (a) inlet of the reactor and (b) middle of the reactor. Black, light gray, and gray dots, respectively, depict vacant sites, $\mathrm{CO}$ species, and oxygen adatoms. At the exit the surface was completely oxygen covered.

The adsorption rate constants are given by $A_{\text {site }} p_{i} / \sqrt{2 \pi m_{i} k_{B} T}$, with $k_{B}$ the Boltzmann constant, and $p_{i}$ and $m_{i}$ the partial pressure and the molecular mass of molecule $i$, respectively, and $A_{\text {site }}$ the area of one adsorption site. For the dissociative adsorption step of oxygen there is another factor $1 / 2$ in this expression because of the equivalence of the atoms in a molecule. ${ }^{18}$ This equation was evaluated at a temperature of $T=500 \mathrm{~K}$ and $A_{\text {site }}=2 \times 10^{-20} \mathrm{~m}^{2}$. Further the fluid velocity $v$ was chosen $1.2 \mathrm{~m} \mathrm{~s}^{-1}$ and $\alpha=15.76 \mathrm{~m}^{-1}$. The partial pressures at the inlet of the reactor were $100.2 \mathrm{~Pa}$ for $\mathrm{CO}, 96.0 \mathrm{~Pa}$ for $\mathrm{O}_{2}$, and no $\mathrm{CO}_{2}$, which gives a $\mathrm{CO} / \mathrm{O}_{2}$ ratio just low enough to prevent $\mathrm{CO}$ poisoning of the catalyst. These numbers and the dimensions of the reactor have been taken from Ref. 24. Further in the reactor $\mathrm{CO}$ reacts to $\mathrm{CO}_{2}$, so that because of the stoichiometry of the reaction the $\mathrm{CO} / \mathrm{O}_{2}$ ratio decreases with as a result that the catalytic surface becomes more oxygen covered (see Fig. 5) which leads to a decrease of the reaction rate. At some point the surface becomes completely covered by oxygen so that the reaction stops and the composition of the species in the gas phase remains constant (see Fig. 6). From this it can be concluded that no thermal equilibrium can be reached due to catalyst poisoning in contradistinction to the mean field model.

\section{NO oxidation at $\mathrm{Pt}(111)$ surface}

We have also modeled a flow reactor wherein the kinetic parameters at the catalytic surface were all estimated from DFT calculations. The oxidation of $\mathrm{NO}$ at a $\mathrm{Pt}(111)$ surface was chosen for this. It has features that are similar to the ZGB model. For instance, the oxygen coverage dominates over that of NO. High coverages of oxygen drive the activation energy of $\mathrm{O}_{2}$ dissociation up, so that this step is rate limiting. ${ }^{25}$ Formation of $\mathrm{NO}_{2}$ is fast because of the modest activation barrier which is relatively insensible to the $\mathrm{O}$ coverage. ${ }^{25}$ In contrast to the very stable $\mathrm{CO}_{2}$ molecule, $\mathrm{NO}_{2}$ formation is reversible however and is an effective $\mathrm{O}$ atom donor to $\mathrm{Pt}(111){ }^{25}$ The model that we have used is also much more complicated than the ZGB model, as there are lateral interactions.

Although it was shown that after adsorption the dissociation of $\mathrm{NO}_{2}$ and $\mathrm{O}_{2}$ take place from bridge sites to fcc hollow sites, for simplicity we modeled this as a single dissociative adsorption step. As a result we could model all reactions with fcc hollow sites in a hexagonal lattice only. In contrast to the grand canonical Monte Carlo simulation in Ref. 26, besides adsorbed $\mathrm{O}$ adatoms we also have NO species at the surface. The system has been modeled by the reaction mechanism in
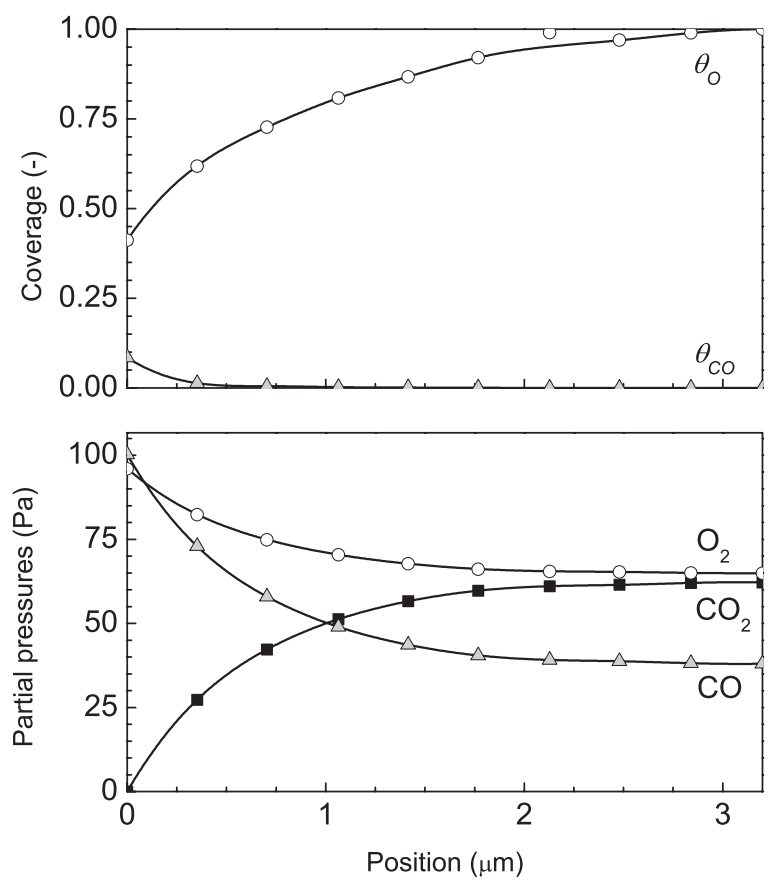

FIG. 6. Coverages and concentrations as a function of the position for the ZGB model as obtained with the gap-tooth scheme. In this scheme we used 401 grid-points and 10 teeth. In the $\mathrm{kMC}$ simulations a $128 \times 128$ square grid was used and a length of $0.1 \mathrm{~s}$. 
the scheme below.

$$
\begin{gathered}
\mathrm{NO}+* \rightleftharpoons \mathrm{NO}_{\mathrm{ads}}, \\
\mathrm{O}_{2}+2 * \rightleftharpoons 2 \mathrm{O}_{\mathrm{ads}}, \\
\mathrm{NO}_{\mathrm{ads}}+\mathrm{O}_{\mathrm{ads}} \rightleftharpoons \mathrm{NO}_{2}+2 * .
\end{gathered}
$$

All elementary reaction steps are reversible and steady state is reached in all $\mathrm{kMC}$ simulations.

The overall reaction rate is slightly exothermic $\left(\Delta H_{0 \mathrm{~K}}\right.$ $=-0.55 \mathrm{eV}^{27}$ ), while the adsorption energies of $\mathrm{NO}, \mathrm{O}_{2}$, and $\mathrm{NO}_{2}$ from DFT calculations in literature are 2.0-2.1, 28,29 $2.4,{ }^{25,26}$ and $1.9^{25} \mathrm{eV}$, respectively. The adsorption energy values of $\mathrm{O}_{2}$ and $\mathrm{NO}_{2}$ corresponded to the energy difference between gas phase species and two neighboring species at the surface. The repulsive energies for nearest neighbors (1$\mathrm{NN}$ ) are $0.2,0.31$, and $0.24 \mathrm{eV}$ for O-O, NO-NO, and NO$\mathrm{O}$ pairs, respectively. The next-nearest neighbor $(2-\mathrm{NN})$ repulsive energies for these pairs are $0.10,0.12$, and $0.10 \mathrm{eV}$, respectively. ${ }^{30}$ The adsorption energies have been corrected via a least-squares method to get proper gas phase heats of reaction. This results in adsorption energies of 1.784, 2.242, and $2.216 \mathrm{eV}$ for $\mathrm{NO}, \mathrm{O}_{2}$, and $\mathrm{NO}_{2}$, respectively. The manybody lateral interactions have been modeled as combinations of 1-NN and 2-NN two-body interactions.

The effect of the lateral interactions on the activation energies have been obtained from a Brønsted-Polanyi relation to relate the shifts in the initial and final state of a reaction to a change in the activation energy $y^{31-33}$

$$
E_{\text {act }}=E_{\text {act }}^{(0)}+\alpha\left(\Delta E-\Delta E^{(0)}\right) .
$$

Here $E_{\text {act }}\left(E_{\text {act }}^{(0)}\right)$ is the activation energy with (without) lateral interactions, and $\Delta E\left(\Delta E^{(0)}\right)$ is the reaction energy with (without) lateral interactions. For $\mathrm{O}_{2}$ and $\mathrm{NO}_{2}$ formation the Brønsted-Polanyi factors $\alpha$ have been estimated to be 1 and 0.2 , respectively. This is appropriate for a late transition state which is typical for (associative) desorption, an early transition typical for a formation. ${ }^{33}$ The influence of the lateral interactions are depicted in Fig. 7. Furthermore we have modeled diffusion of oxygen and NO over bridge sites with activation energies of $0.56^{30,34}$ and $0.23 \mathrm{eV},{ }^{29,30,35}$ respectively, which were influenced by lateral interactions with a Brønstead-Polanyi factor of 0.5. All prefactors have been taken to be $10^{13}$, while the adsorption rates have been, similarly as in the $\mathrm{CO}$ oxidation section, calculated with $\left(A_{\text {site }} p_{i} / \sqrt{2 \pi m_{i} k_{B} T}\right) \exp \left[-E_{i \text {,act }} / k_{B} T\right]$. For the dissociative adsorption step of $\mathrm{NO}_{2}$ this expression has been statistically corrected by a factor $1 / 3$ for the number of neighboring catalytic sites at a hexagonal grid. ${ }^{18}$ To reduce the computation time the prefactors of diffusion have been lowered by a few orders of magnitude, such that diffusion is still fast enough to equilibrate the surface between adsorption/desorption and conversion steps.

In the $\mathrm{kMC}$ simulations a $64 \times 64$ square grid and a simulation length of $1000 \mathrm{~s}$ has been used. The reactor is again discretized in 401 grid points with 20 evenly spaced teeth. At

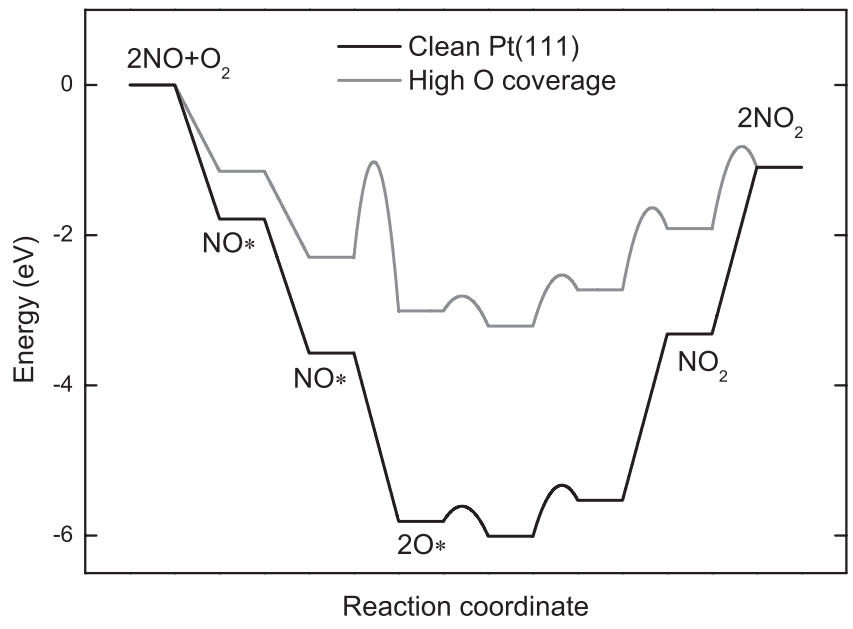

FIG. 7. Reaction diagram of $\mathrm{NO}$ oxidation at a $\mathrm{Pt}(111)$ surface. At a clean surface (black line) two NO species adsorb and $\mathrm{O}_{2}$ adsorbs dissociatively. This is followed by two diffusive processes resulting in the two activation barriers to the right of 20*. The first is from the separation of the oxygen atoms. The second is the formation of an NO-O neighboring pair. In the rate limiting step two $\mathrm{NO}_{2}$ species are formed. When there is more oxygen at the surface (gray line), repulsive interactions arise that decrease the activation barriers significantly after which $\mathrm{O}_{2}$ dissociation becomes the rate limiting step.

the inlet of the reactor $1000 \mathrm{ppm}$ of $\mathrm{NO}$ is fed to the reactor in $0.05 \mathrm{~atm}$ of oxygen. At the inlet of the reactor the surface is covered by $\sim 0.07$ monolayer (ML) of $\mathrm{NO}$ and $\sim 0.37 \mathrm{ML}$ of $\mathrm{O}$ adatoms. Further in the reactor $\mathrm{NO}$ is converted to $\mathrm{NO}_{2}$

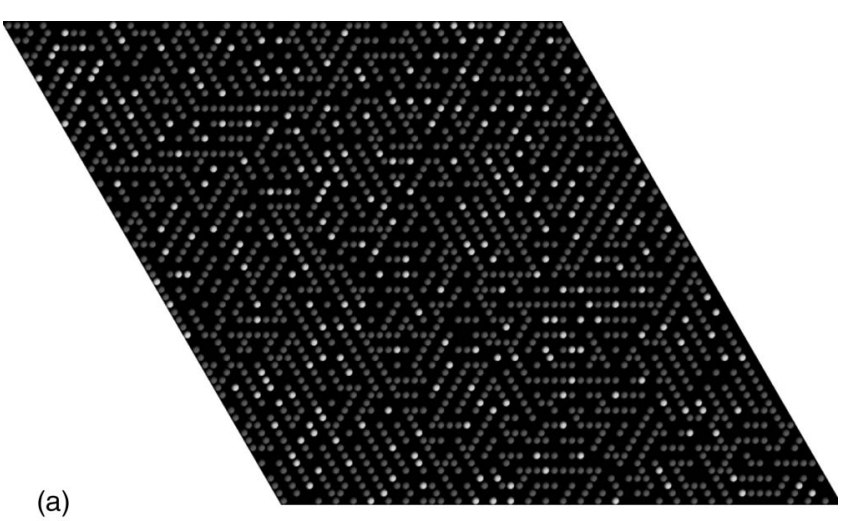

(b)

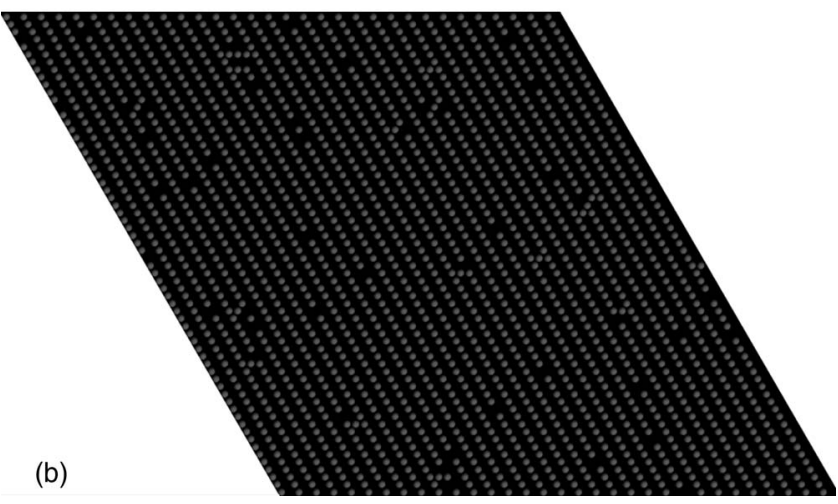

FIG. 8. Configurations of adsorbates at the catalytic surface at the (a) inlet of the reactor and (b) exit of the reactor. Black, light gray, and gray dots, respectively, depict vacant sites, $\mathrm{NO}$ species, and oxygen adatoms. 

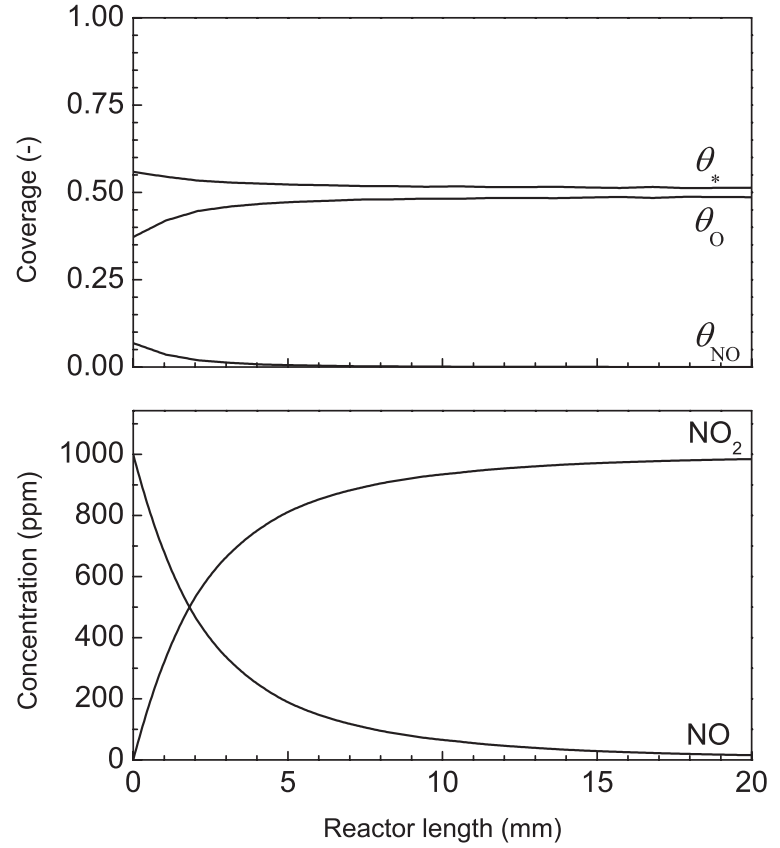

FIG. 9. Coverages and concentrations as a function of the position for the NO oxidation on $\mathrm{Pt}(111)$ model as obtained with the gap-tooth scheme. In this scheme we used 401 grid-points and 20 teeth. In the kMC simulations a $64 \times 64$ square grid was used and 1000 time steps of $1 \mathrm{~s}$ each.

with the result that more oxygen is donated to the surface and its coverage decreases because of the lower concentration of NO. At the exit an oxygen coverage of $\sim 0.5 \mathrm{ML}$ is reached while the NO coverage is $\sim 0 \mathrm{ML}$. The configuration of the adsorbates at the surface is depicted in Fig. 8 at the inlet and exit of the reactor (see also Fig. 9). In contrast to the ZGB model, although at the exit no NO is adsorbed, the surface is still reactive, i.e., when NO adsorbs it immediately reacts to the product and desorbs. Reversible reaction steps and surface diffusion caused defects in the structure, at which NO can adsorb and is almost immediately converted to $\mathrm{NO}_{2}$ in the gas phase.

Although we did not take all details into account (e.g., adsorption and dissociation in separate steps), most features of our model were essentially the same as in the Refs. 18, 25-30, 34, 35. Quantitative improvements are expected to be obtained by taking three-body lateral interactions into account and by modeling adsorption and dissociation of $\mathrm{O}_{2}$ and $\mathrm{NO}_{2}$ in separate steps. The most important result is however that we showed that with a realistic reaction model we were able to perform a reaction simulations taking just a few hours on a regular desktop computer.

\section{CONCLUSIONS}

In this work we have shown that we have successfully implemented a method to model simple flow reactors with realistic reaction mechanisms in an acceptable computer time. This method is applicable for reactors at steady state, and does not yet account for nonlinear effects such as moving fronts, oscillations, and bistability. To deal with these effects more advanced techniques are required, e.g., one should can think of using dynamic teeth positions and of building a data base wherein $\mathrm{kMC}$ results are stored. Our approach can potentially be extended by incorporating transport limitations in more sophisticated CFD simulations. The method in our work is an extension of the method in the work of Majumder et al. ${ }^{15}$ wherein a gap-tooth scheme was used. The regions between teeth however have been treated sequentially so that the required number of $\mathrm{kMC}$ simulations was reduced. The output of the kMC simulations in our approach is corrected to obey mass conservation by a least-squares method based on a stoichiometry matrix.

Our method has been validated by considering a unimolecular reaction of which the elementary reaction steps take place on a single site and wherein there are no lateral interactions. In this case the kMC results could be compared to exact analytical results. These should be the same except for errors caused by the stochastic nature of $\mathrm{kMC}$ and the discretization of the reactor. The performance of the gaptooth scheme has been studied by varying the grid density and the number of teeth from which it can be seen that the number of kMC simulations can be reduced to $10-15$ without influencing the calculation error significantly. Potentially, by not evenly spacing the teeth, this number might be further reduced.

We have applied the method to simulate a CO oxidation reactor in which the mechanism has been modeled by the ZGB model, and to a reactor with NO oxidation on a $\operatorname{Pt}(111)$ surface. These simulations have shown some interesting similarities and differences. In both models the coverage of oxygen dominates at the surface while $\mathrm{CO}$ and $\mathrm{NO}$ are rapidly converted to the product. At the inlet of the reactor $\mathrm{CO}$ and NO have relatively high concentrations, which results in the highest coverages there of these species and of the vacant sites. Further in the reactor $\mathrm{CO}$ and NO are converted to the products so that their coverages decrease and the oxygen coverage increases. The differences lie in the lateral interactions and reversible reaction steps in the NO oxidation mechanism. In the ZGB model for $\mathrm{CO}$ oxidation the catalyst poisons. Due to lateral interactions in the NO oxidation model the catalyst becomes less active, but does not poison. Qualitative features of the NO oxidation model (e.g., an increased oxygen coverage changes the rate limiting step from $\mathrm{NO}_{2}$ formation step to $\mathrm{O}_{2}$ dissociation) are consistent with literature. We expect that a many-body interaction description between adsorbates and separated adsorption/dissociation steps will also improve the quantitative agreement with experiments.

${ }^{1}$ R. A. van Santen, M. Neurock, and S. G. Shetty, Chem. Rev. 110, 2005 (2010).

${ }^{2}$ M. K. Sabbe, M.-F. Reyniers, and K. Reuters, Catal. Sci. Technol. 2, 2010 (2012).

${ }^{3}$ S. Raimondeau and D. G. Vlachos, Chem. Eng. J. 90, 3 (2002).

${ }^{4}$ T. J. Pricer, M. J. Kushner, and R. C. Alkire, J. Electrochem. Soc. 149, C396 (2002).

${ }^{5}$ T. J. Pricer, M. J. Kushner, and R. C. Alkire, J. Electrochem. Soc. 149, C406 (2002)

${ }^{6}$ T. O. Drews, E. G. Webb, D. L. Ma, J. Alameda, R. D. Braatz, and R. C. Alkire, AIChE J. 50, 226 (2004).

${ }^{7}$ E. Rusli, T. O. Drews, and R. D. Braatz, Chem. Eng. Sci. 59, 5607 (2004). ${ }^{8}$ Z. Zheng, R. M. Stephens, R. D. Braatz, R. C. Alkire, and L. R. Petzold, J. Comp. Phys. 227, 5184 (2008).

${ }^{9}$ T. C. Castonguay and F. Wang, J. Chem. Phys. 128, 124706 (2008).

${ }^{10}$ N. Hansen, R. Krishna, J. M. van Baten, A. T. Bell, and F. J. Keil, J. Phys. Chem. C 113, 235 (2009). 
${ }^{11}$ S. Matera and K. Reuter, Catal. Lett. 133, 156 (2009).

${ }^{12}$ S. Matera and K. Reuter, Phys. Rev. B 82, 085446 (2010).

${ }^{13}$ N. Hansen, R. Krishna, J. M. van Baten, A. T. Bell, and F. J. Keil, Chem. Eng. Sci. 65, 2472 (2010).

${ }^{14}$ F. J. Keil, Top. Curr. Chem. 307, 69 (2012).

${ }^{15}$ D. Majumder and L. J. Broadbelt, AIChE J. 52, 4214 (2006).

${ }^{16}$ S. Matera and K. Reuter, J. Catal. 295, 261 (2012).

${ }^{17}$ I. G. Kevrekidis, C. W. Gear, J. M. Hyman, P. G. Kevrekidis, O. Runborg, and C. Theodoropoulos, Commun. Math. Sci. 1, 715 (2003).

${ }^{18}$ A. P. J. Jansen, Introduction to Kinetic Monte Carlo Simulations of Surface Reactions, Lecture Notes in Physics Vol. 856 (Springer, Heidelberg, 2012).

${ }^{19}$ W. H. Press, B. P. Flannery, S. A. Teukolsky, and W. T. Vetterling, Numerical Recipes. The Art of Scientific Computing (Cambridge University Press, Cambridge, 1989).

${ }^{20}$ A. P. de Wasch and G. F. Froment, Chem. Eng. Sci. 26, 629 (1971).

${ }^{21}$ D. A. McQuarrie, Statistical Mechanics (Harper, New York, 1976).

${ }^{22}$ H. S. Fogler, Elements of Chemical Reaction Engineering (Prentice-Hall, Englewood-Cliffs, 2005).
${ }^{23}$ R. M. Ziff, E. Gulari, and Y. Barshad, Phys. Rev. Lett. 56, 2553 (1986).

${ }^{24}$ G. Chen, Q. Yuan, H. Li, and S. Li, Chem. Eng. J. 101, 101 (2004).

${ }^{25}$ R. B. Getman, W. F. Schneider, A. D. Smeltz, W. N. Delgass, and F. H. Ribeiro, Phys. Rev. Lett. 102, 076101 (2009).

${ }^{26}$ C. Wu, D. J. Schmidt, C. Wolverton, and W. F. Schneider, J. Catal. 286, 88 (2012).

${ }^{27}$ M. W. Chase, Jr., NIST-JANAF Themochemical Tables, 4th ed., Monograph 9 (NIST, Gaithersburg, 1998).

${ }^{28}$ H. Tang and B. L. Trout, J. Phys. Chem. B 109, 17630 (2005).

${ }^{29}$ A. Bogicevic and K. C. Hass, Surf. Sci. 506, L237 (2002).

${ }^{30}$ S. Ovesson, B. I. Lundqvist, W. F. Schneider, and A. Bogicevic, Phys. Rev. B 71, 115406 (2005).

${ }^{31}$ J. N. Brønsted, Chem. Rev. 5, 231 (1928).

${ }^{32}$ M. G. Evans and M. Polanyi, Trans. Faraday Soc. 34, 11 (1938).

${ }^{33}$ C. G. M. Hermse, and A. P. J. Jansen, in Catalysis, edited by J. J. Spivey and K. M. Dooley (Royal Society of Chemistry, London, 2006), Vol. 19.

${ }^{34}$ W. K. Offermans, Ph.D. dissertation, Eindhoven University of Technology, Eindhoven, 2007.

${ }^{35}$ Q. Ge and D. A. King, Chem. Phys. Lett. 285, 15 (1998). 